\title{
Engineering of $\beta$-glucosidase Bgl15 with simultaneously enhanced glucose tolerance and thermostability to improve its performance in high-solid cellulose hydrolysis
}

Lichuang $\mathrm{Cao}^{1}{ }^{\S}$, Ran Chen ${ }^{2}$, Xin Huang ${ }^{1}$, Shuifeng $\mathrm{Li}^{1}$, Sufang Zhang, ${ }^{1}$ Xiangpeng Yang, ${ }^{1}$ Zongmin Qin, ${ }^{1}$ Wei Kong, ${ }^{1}$ Wei Xie ${ }^{2 *}$, Yuhuan Liu ${ }^{1 *}$

${ }^{1}$ School of Life Sciences, Institute of Aquatic Economic Animals and Guangdong Provincial Key Laboratory for Aquatic Economic Animals, National Engineering Center for Marine Biotechnology of South China Sea, ${ }^{2}$ Ministry of Education Key Laboratory of Gene Function and Regulation, State Key Laboratory for Biocontrol, Sun Yat-Sen University, Guangzhou, 510275, P. R. China

*Correspondence should be addressed to: Wei Xie (Email:

xiewei6@mail.sysu.edu.cn.) or Yuhuan Liu (E-mail: 1sslyh@mail.sysu.edu.cn).

Tel: 86-20-84113712, Fax: 86-20-84036215.

$\S$ These authors contributed equally to this work. 


\section{Supporting Information}

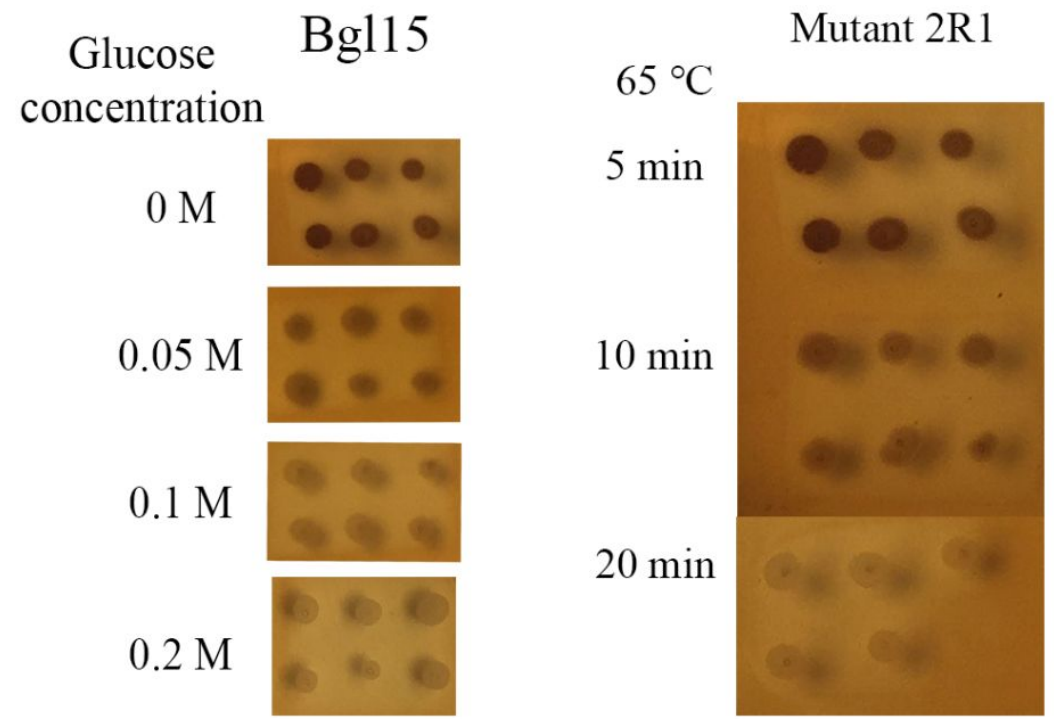

Figure S1. Optimization of the screening conditions. Optimization of the screening condition for the first (left) and third (right) round of screening. 

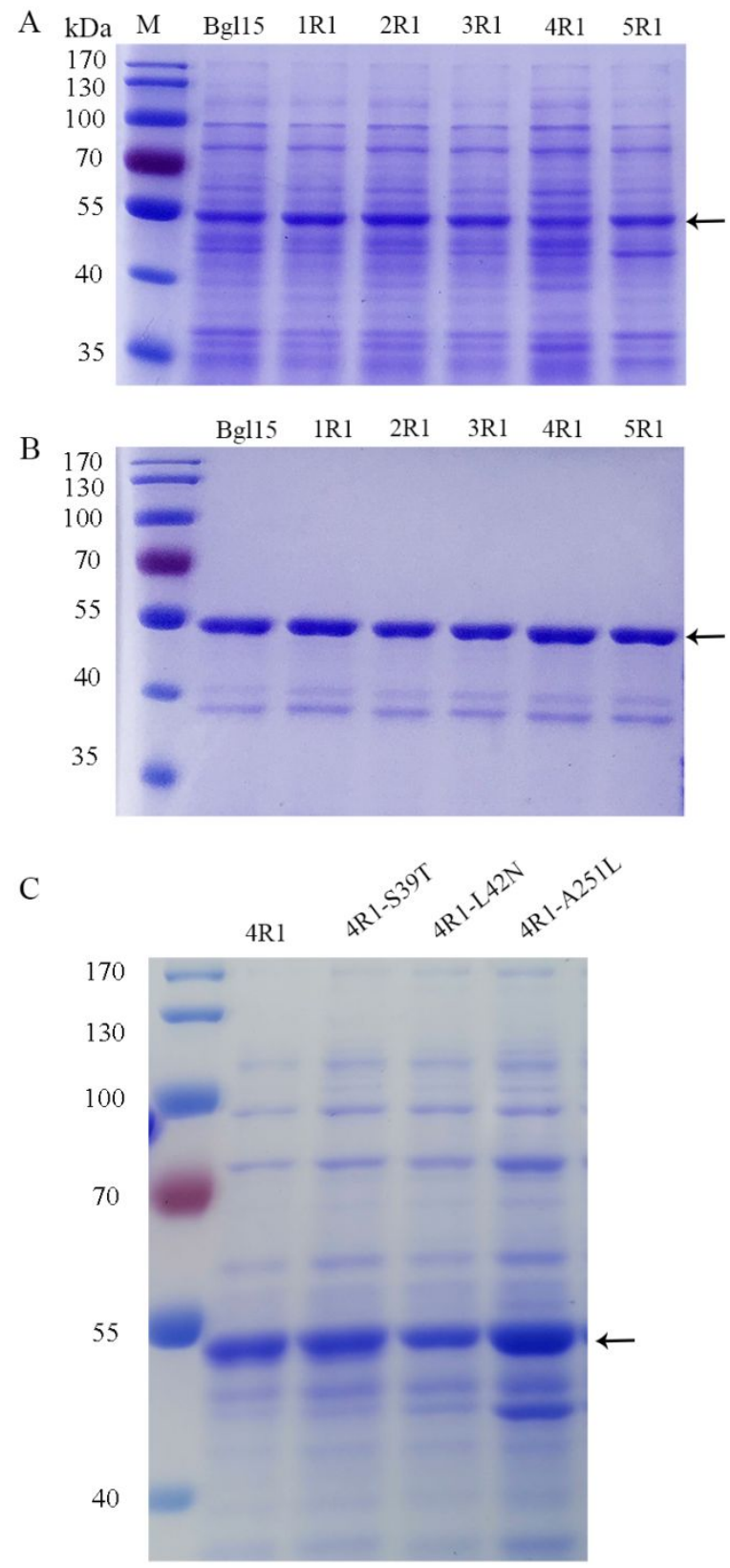

Figure S2. SDS-PAGE analysis of the supernatants of cell lysate $(A, C)$ and the purified recombinant proteins (B). In (A), the sample amount of mutant 5R1 was half of Bgl15 and other mutants. In (B), the sample amount was $2 \mu \mathrm{g}$ for each lane. In (C), the same volume of samples was used. Lane M, standard protein molecular mass marker (Thermo Fisher Scientific, sizes in kilodaltons are indicated on the left). The 
specific bands of the target protein were marked by an arrow.

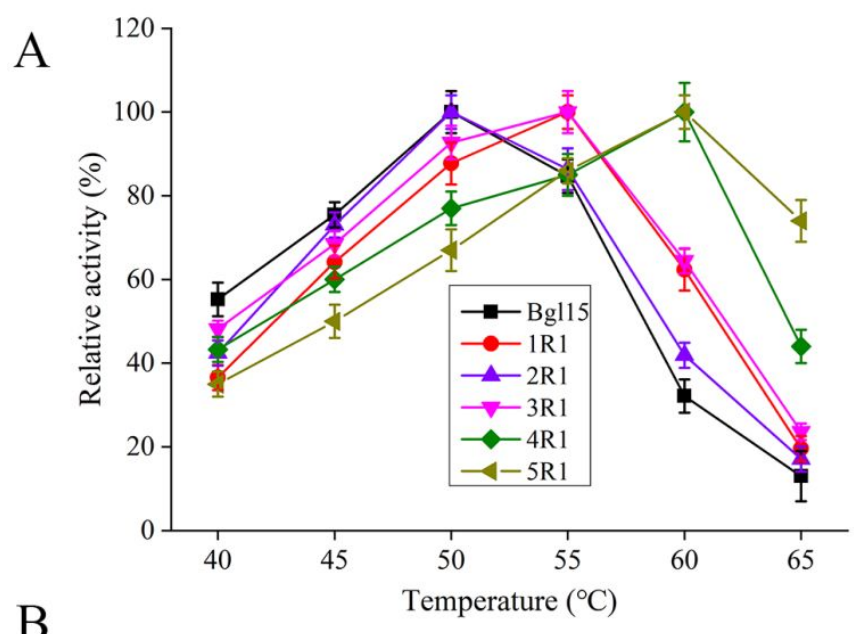

B

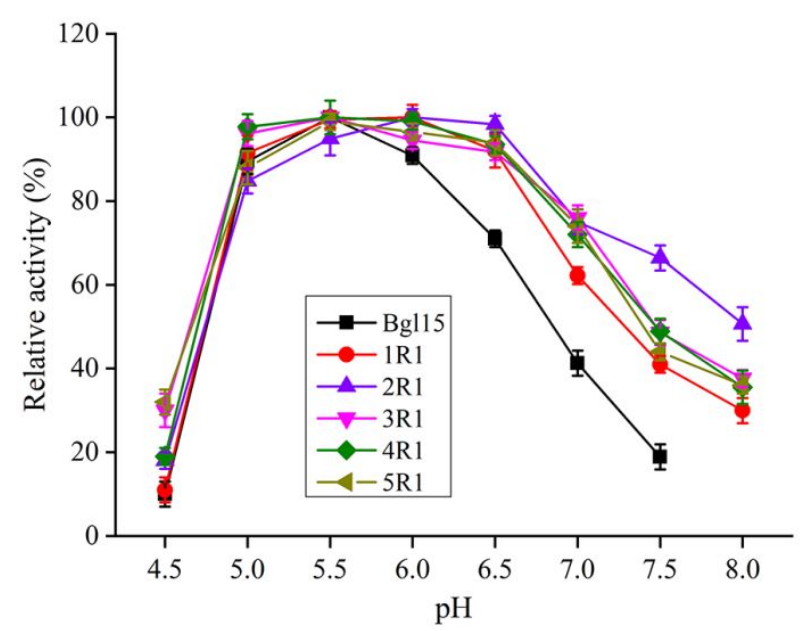

Figure S3. Optimal temperatures (A) and optimal pH values (B) of Bgl15 and its

mutants. (A) Optimal temperature was assayed in $\mathrm{pH} 6.0$ buffer. (B) Optimal pH was assayed at $50{ }^{\circ} \mathrm{C}$. Data points are the average of triplicate measurements, and error bars represent standard deviation. 


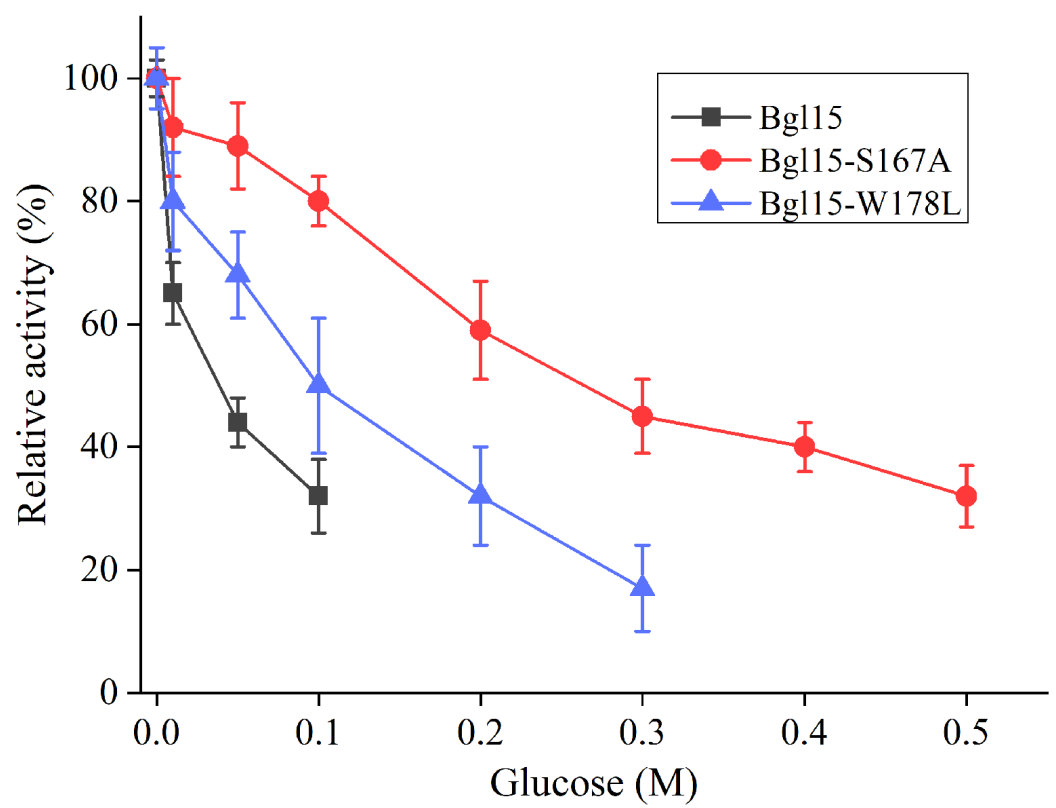

Figure S4. Glucose tolerance of single point mutants Bgl15-S167A and

Bgl15-W178L. Reactions were performed at $50^{\circ} \mathrm{C}$ and $\mathrm{pH} 6.0$. The $I C_{50}$ values are 0.04 M (Bgl15), 0.25 M (Bgl15-S167A) and 0.1 M (Bg115-W178L). 


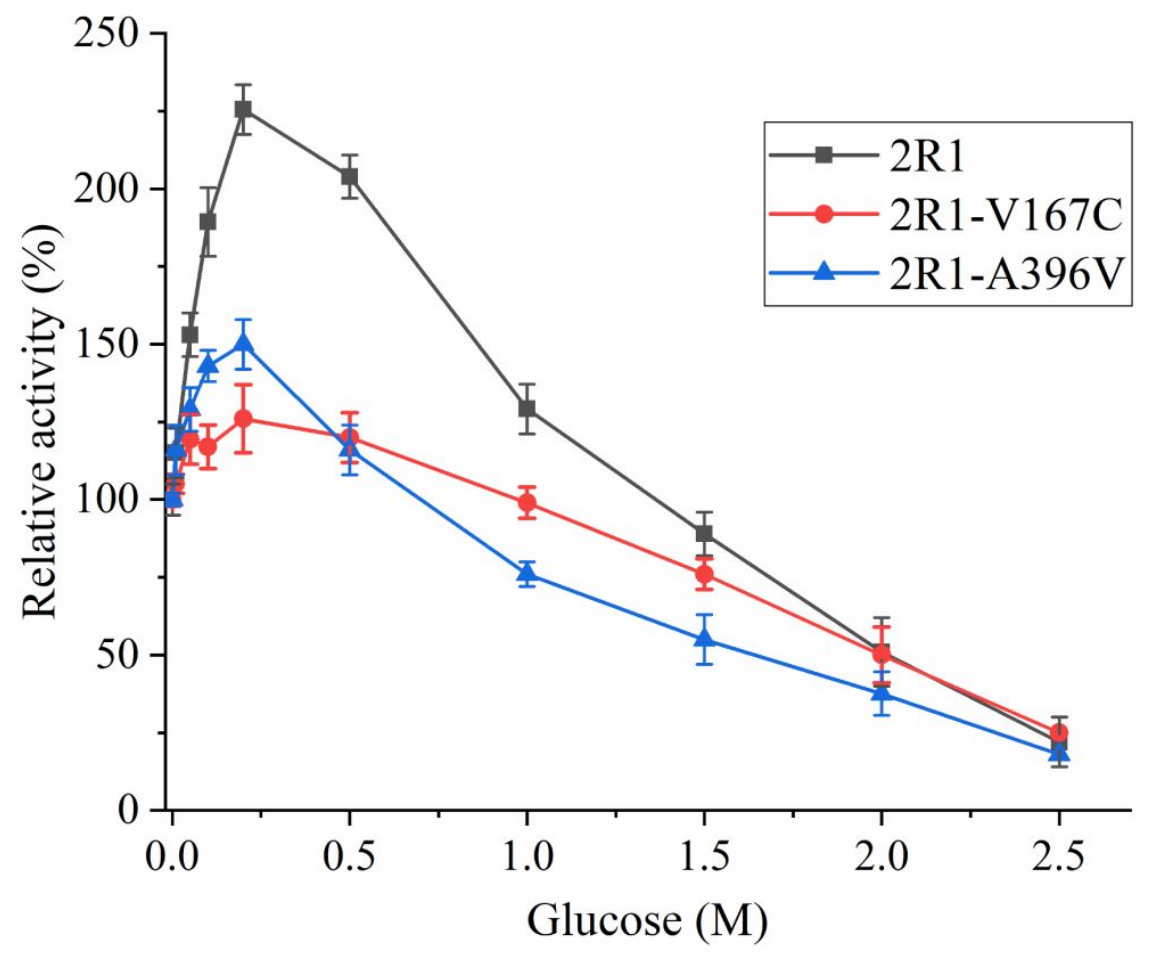

Figure S5. Glucose tolerance of mutants 2R1-V167C and 2R1-A396V. Reactions were performed at $50{ }^{\circ} \mathrm{C}$ and $\mathrm{pH} 6.0$. The $I C_{50}$ values are $2.1 \mathrm{M}(2 \mathrm{R} 1), 2.1 \mathrm{M}$ (2R1-V167C) and 1.9 M (2R1-A396V). The maximal stimulation levels are 2.25-fold (2R1), 1.25-fold (2R1-V167C) and 1.5-fold (2R1-A396V). Data points are the average of triplicate measurements, and error bars represent standard deviation. 


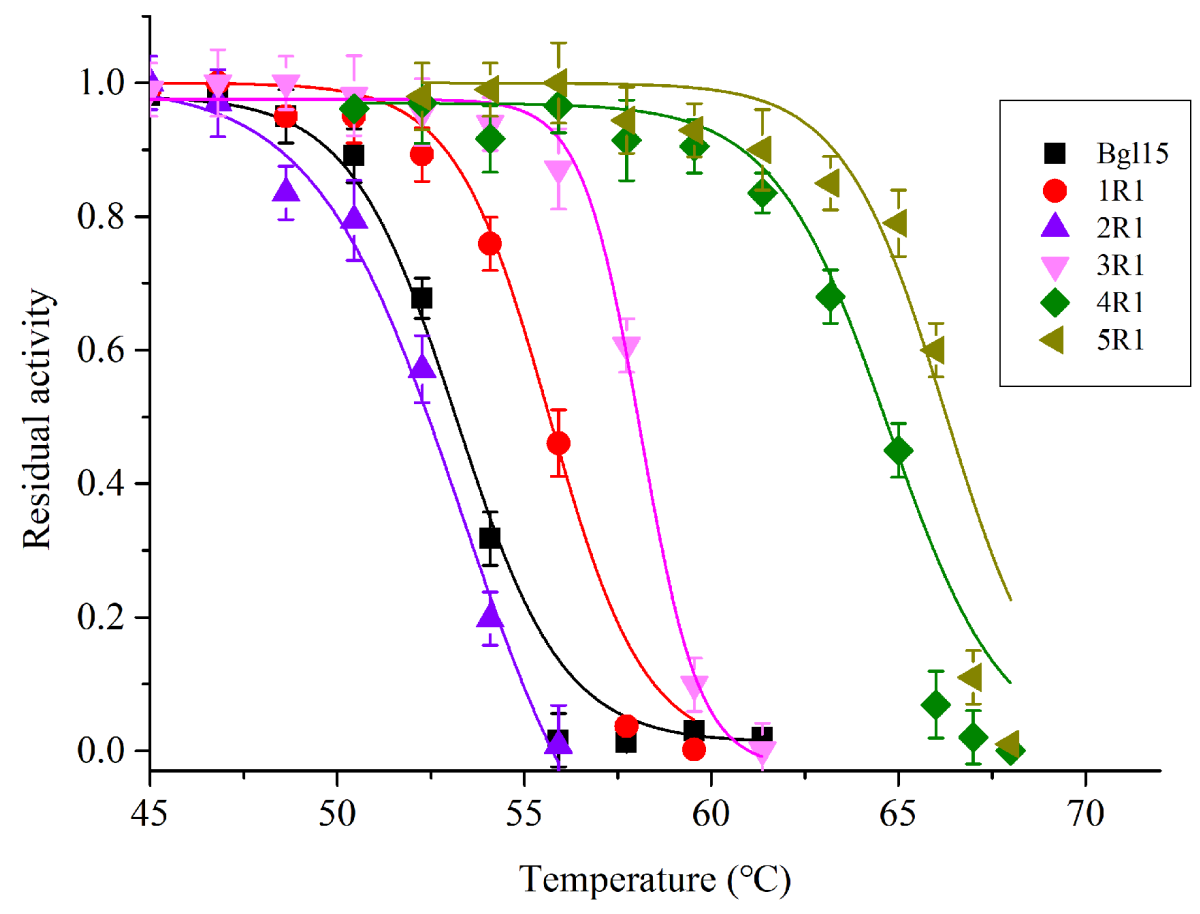

Figure S6. Thermal inactivation curves of the mutants in this study. The $T_{50}$ values are 53.2 (Bg115), 55.8 (1R2), 52.9 (2R1), 58.1 (3R1), $64.4(4 \mathrm{R} 1)$ and 66.3 $(5 \mathrm{R} 1){ }^{\circ} \mathrm{C}$. 
QED88370.1

OED 88370 AKH41028.1 AAU92142.1 AFU63315.1 QAB08112.1 HV538882.1 BAV69317.1 Q60026 AKP 4 5355. 1 WP_013298275.1

ASI 24660.1 AOW70162.1 AFS 69459 . 1 AFO 36783.1 AFQ36783.1 AGS 46809.1 AGS 03809.1 $\mathrm{AB} Q 46970$. 1 ASI13273.1 APG42678. ABK51908.1 QAB08113.1 QBC98235.1 ACM59590.1 ADD96762.1 AXR85509.1 ABR73190.1 EHJ97046.1 HV348683.1 HV348683. AHG2 3303. BAB 91145.1 BAI50022.1 AFD 33364.1 BAU51446.1 BAA74959.1 AMP 46472.1 AII 80277 . 1 XP_011393758.

AFK08794.1 AKN80694.1 CAA94187.1 OCG75868. 1 CCA60742.1 YP_004660190.1 CDḠ15325.1

CDA15325. AAL80197.1

OED 88370.1

OED 88370.1

AKH4 1028 . 1

AAU 92142.1

AFU63315.1

OAB08112.1

HV538882.1

BAV69317.1

BAV69317

AKP 45355. 1

AKP 45355.1

ASL 24660.1

AOW70162.1

AFS 69459 .

AFQ36783.1

WP_013486910.1

AGS 46809.1

WP_039812498.1

ABQ46970.

ASI13273.1

APG4 2678. 1

ABK51908. 1

OAB08113.1

QAB 98235.1

QBC98235.

.

ADD96762.1

AXR85509.1

ABR7 3190.1

EHJ97046.1

HV348683.1

AHG23303.

BAB91145.

BAI50022.

AFD 33364 . 1

BAU51446.1

BAA7 7959.1

AMP 46472 .

AII80277.1

XP_011393758.

AFK 08794.1

AKN80694.

AKN80694.1

CAA94187.

QCG75868.1

CCA60742.1

YP_004660190.1

CDG15325. 1

AAL80197.1

consensus $>50$ т т $\underset{10}{\stackrel{\beta 1}{\longrightarrow}} \stackrel{\alpha e_{20}^{1}}{\longrightarrow}$

ee

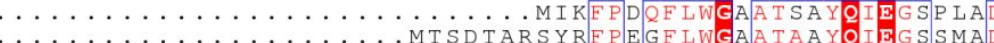

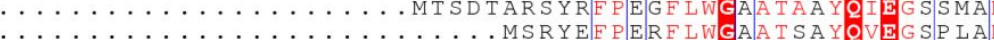

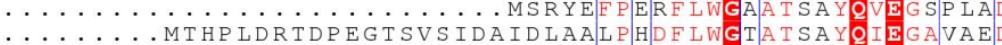

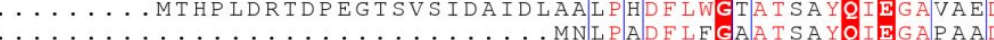

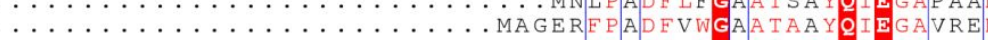

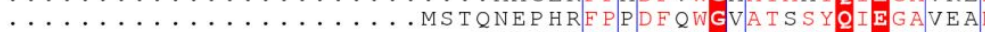

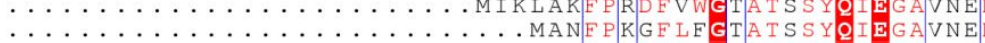

MSDFNKDFLFGVATASYOVFGAYNE

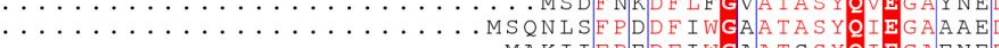

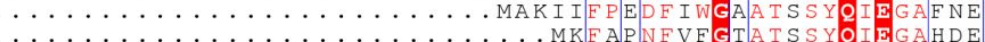

MSE

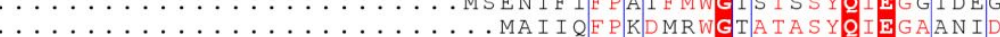

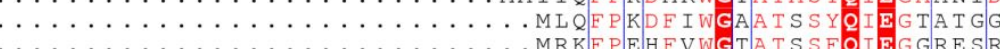

(1) GRES

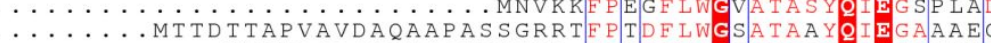

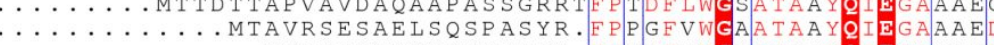

$\ldots \ldots \ldots \ldots$ M Q IEERDQVESRPT LR. F P D RF VWGVA T SAY I EGAVAED

$\ldots \ldots \ldots$ MTAHETQAQIREPATAP....................

$\ldots . . . . V$ VTSQSTASLGQTEEEPQRDIR...FP Q DFVWGAATASEQVEGATTAD

$\ldots \ldots \ldots \ldots \ldots \ldots \ldots$.

$\ldots \ldots \ldots \ldots \ldots \ldots \ldots \ldots$

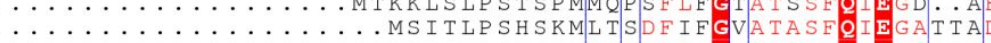

$\ldots \ldots \ldots \ldots \ldots \ldots \ldots \ldots$ M DHKALAARF P G F LF GVA TASF (1)

.

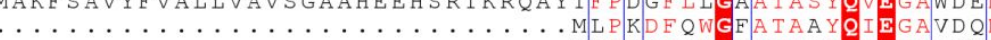

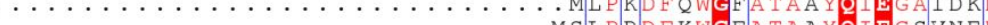

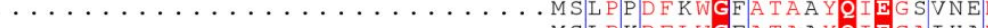

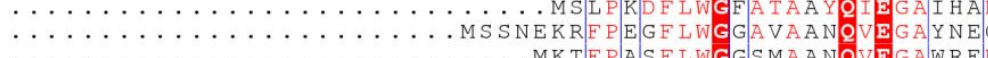
MKT T PAS S LWGGS MAANQVEGAWRED

RE DGELLTATSS

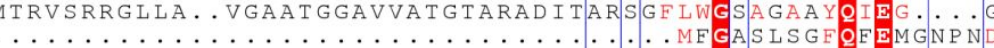
MFP

MVKFP KGFMWGISMAA

$\ldots \ldots \ldots \ldots \ldots \ldots$

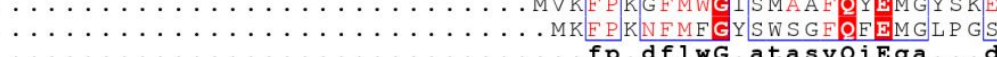

$\eta 1$

elee.e.

$\alpha 3$

..e 30

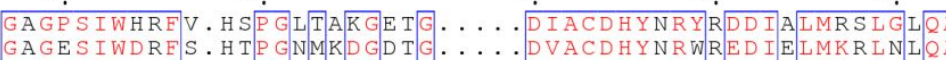
GAGPSNWHREC.ROPGRT L NGDTG..... DTACDHYRRFREDVALMKALGLS

$\ldots$ GRSPSIWDTES. HTPGIIDNGDHG . . . D D DACDHYHRWRED IALMRRLGT TA

$\ldots$ GRLPSIWDDEC.RVPGAIAGGDTG..... DVACDHYHRWREDVALMRRLGIDA

GRGVSIWDTES.HTPGIIADGTTG..... DVACDSYHRYGEDIGILNA G GM

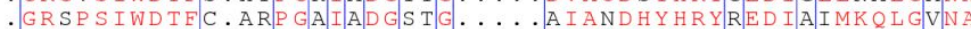

GRTPSIWDTFS.KTEGKTYKGHTG $\cdots . . .$. DVACDHYHRYKEDVEILKEIGVK

.

................

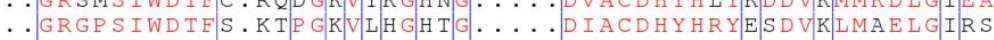

$\ldots$ GKGESIWDRES.HTPGKIENGDTG..... D I ACDHYH LYREDIEIMKEIGIRS

.. GRTPSIWDTEC. DTDGKVFEKHNG.... DVACDHYHRFEEDIQH I K L LGVDT

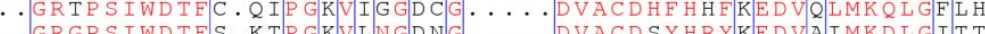

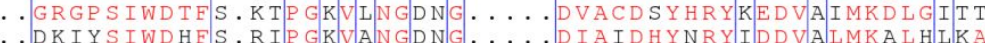

$\ldots$ DKIYSIWDHES.RIP GKVANGDNG.... D I A I DHYNRYID D VA L MKA L H L KA

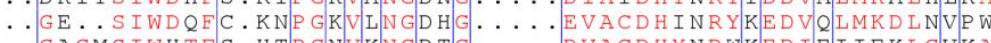

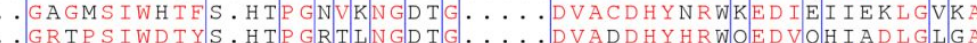

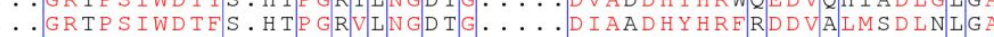

$\ldots$ GRGPSIWDTES.HTP GKVVGGDTG..... DVAADHYHRYVGDVRMADLGVIS

. . GRGRS IWDTEC.DTP GAVIGGDTA. . . . A A AVDHYHRYREDVALMA G L G L

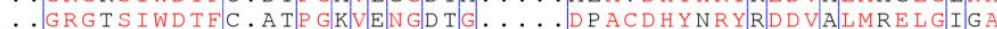

$\cdots$ GKGESIWDRFT. HOKGNI Y H H . .........

..................

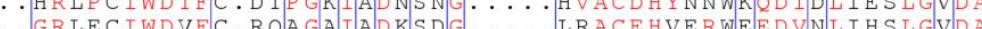

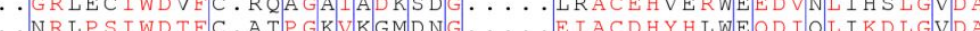

$\cdots$ NRLP SIWD TEC. A T P GKVKGMDNG..... E I ACD HYH LWEQD I Q L I K D L GVD

..GRKPSIWDAFC.NMPGHVE GRHNG..... DVACDHYNRWEDD L D L I KEMGVEA

$\ldots$ GRGKDIWGDYC.S I P G I IF D N H NG..... D KACEHYYRYKEDVAIMKEMGIKA

.. GRGP SVWD LMC.RWPGKVSRG H SG. . . . EVACDHYHRYAEDVRLAREVGAKA

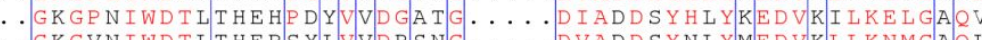

. GKGVNIWDTLTHERSY LVVDRS NG. . . . . DVAD D SYNLYMEDVKLLKNMGA $Q$

GKGVNIWDTLTHERP Q LVVDHSSG. . . . . DVADDSYHLYTEDVKLLKNMGAQV

$\ldots$ GKTSSIWDTQTHDKNYLIADHTT G. . . . D I ACD SYHKYDVDVQMLRD L GVDE

$\ldots$ GRGPSIWDTEC. AQP GKIADGSSG.....VTACDSYNRTAEDIAILKS LGAKS

$\ldots$ GRGPSIWDTEC. A IP GKIADGTSG.....VVTACDSYNRTAEDIALLKS LGAKS

.. GRGPSIWDTEC. A IPGKIADGSSG..... AVACDSYKRTKEDIALLKELGANS

$\ldots$ GRGPSIWDTEC.NIPGIIADGSSG.....A AVACD SYNRTKEDIDILKSIGATA

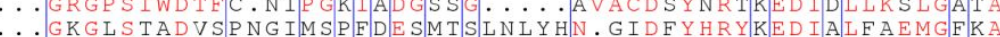

..GKG L S TADVSPNG IMS P F DESMT S L N L Y H N. G I D F Y HRYKED I A LFAEMGFKA

NVWSDWW. .YWAEKGKL. . . P PAG...... KACNSWELYEKDLELMAGLGYA NVASDLW...VVEHVQP TI I REASG...... DAVDAYHRVF D D IAILAAS L GF NA

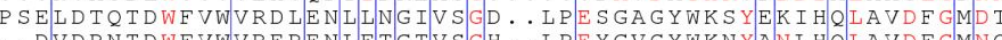
.. DVDPNTDWFVWVREP EN L F T GTVSG H . L L E E GVGYWKNYANL HQ LAVDEGMNC .. AID QN SDWY LWLHNE ENKKKR G IVSGD . VP EMGP GYWDLYKVDHDWAEWLG LN T

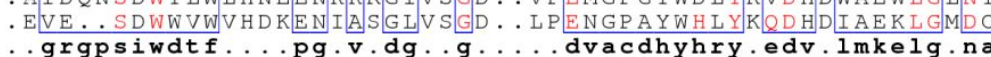



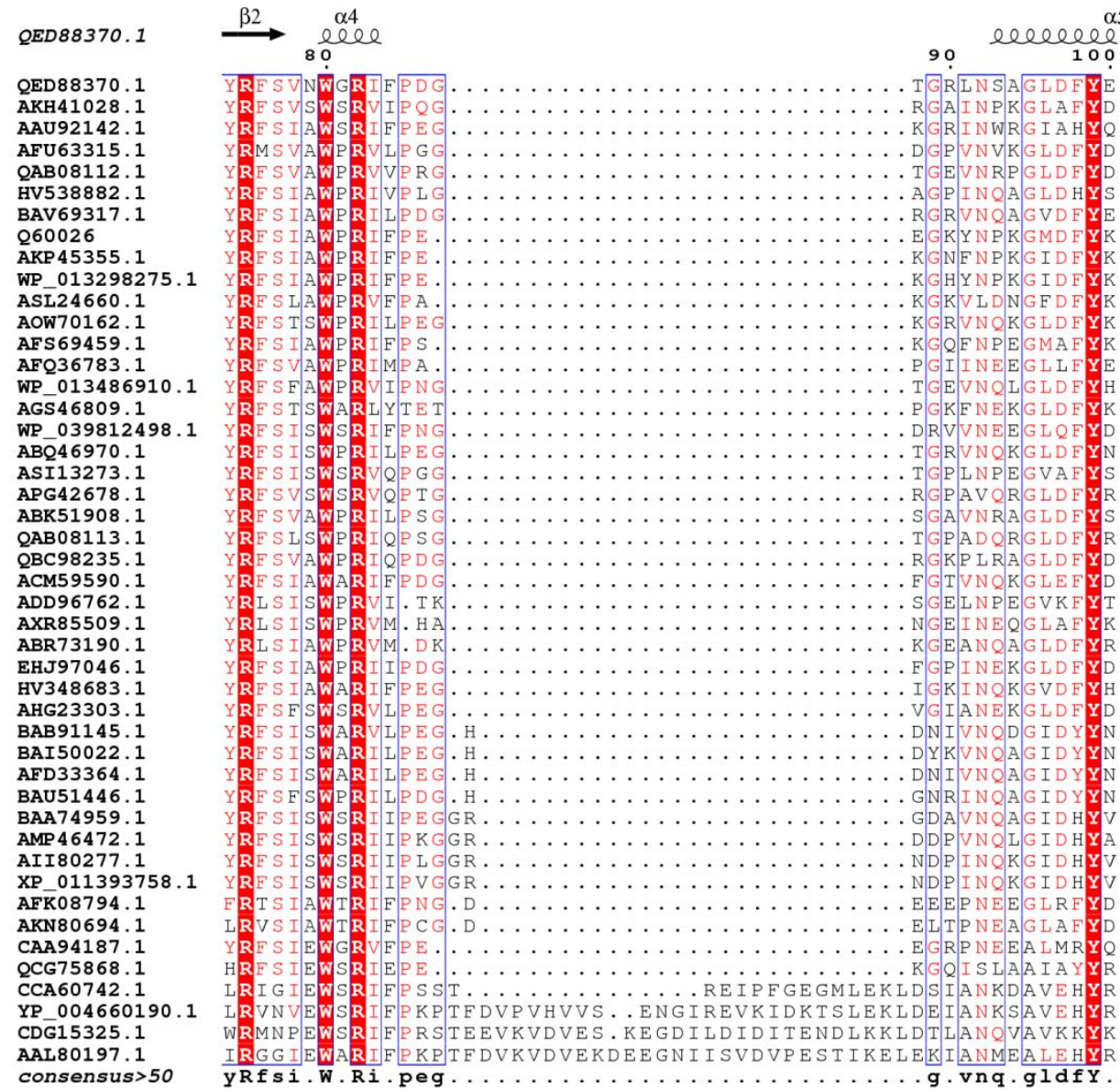

OED 88370

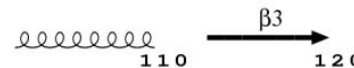

eele

$\eta 2$

QED 88370.1

AKH4 1028. 1

AAU92142.1

AFU63315. 1

QAB08112.1

HV538882.1

BAV69317.1

060026

AKP 45355. 1

WP_013298275.1

ASL 24660 .

AOW70162.1

AFS 69459.1

AFO36783.1

WP_013486910.

AGS 46809.1

WP_039812498.1

$\mathrm{ABO} 46970.1$

ABQ 46970.1

APG42678.1

APG52678.1

ABK51908.1

QAB 08113.1

QBC98235.

ACM59590.1

ADD 96762.1

AXR85509.1

ABR73190.

EHJ97046.1

HV348683.1

AHG23303.1

BAB 91145.1

BAI50022.1

AFD 33364.1

BAU51446.1

BAA7 4959.1

AMP 46472 . 1

AII80277.1

XP_011393758.

AFK̄0 8794.1

AKN80694.1

CAA94187. 1

QCG75868.

CCA60742.1

YP_004660190.1

CDG'15325.

AAL 80197 . 1

consensus $>50$

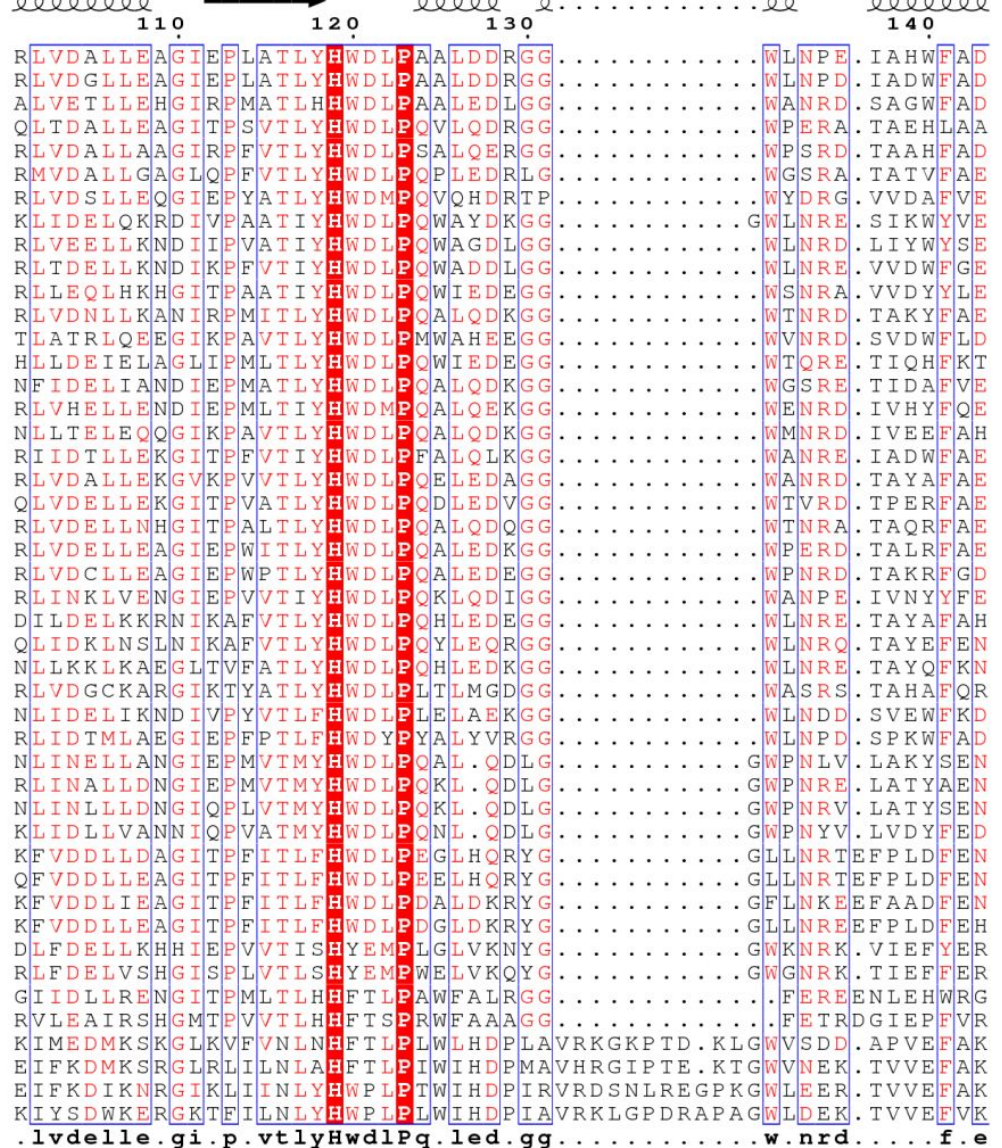




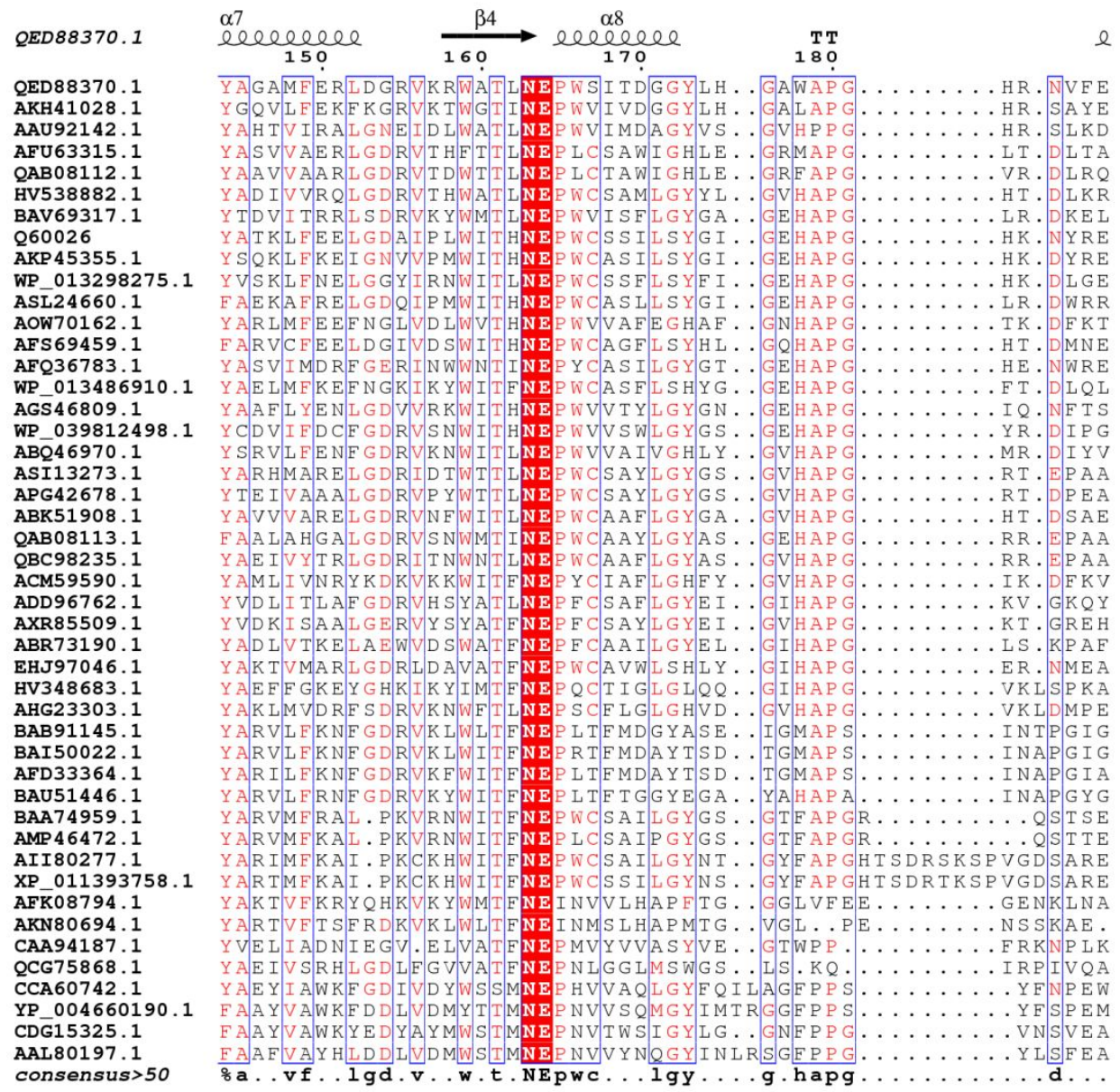

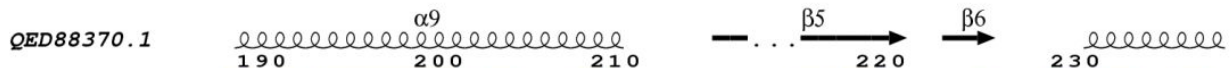
QED88370.1 APIASRNLMLAHGAVQRYRQAGKH..EI...GLVVNIEPKYPAS. ESD SDRNAAA AKH41028.1

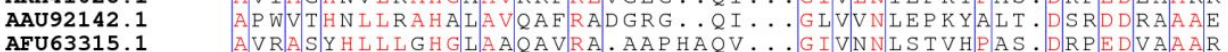

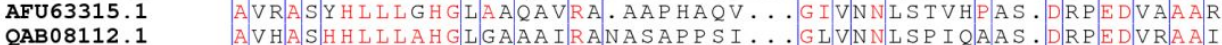
HV538882.1 GLEASHNLLLGGLAVQAMRAAAPQPLQI... GIVINLTPTYRAS. DSPEDVAAAR BAV69317.1 A L IAAHH I L SHGEAVKAEREMNIKGSKI... G ITINITPAYPAS. EKEEDKLAAQ

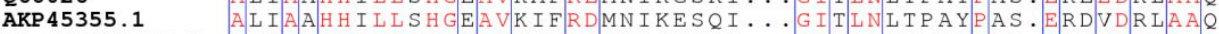
WP_013298275.1 AVLVSHNLLLAHGKAVEIFRD INS S D K I . . . G I T L N L NEVFPA T.DSPEDKAAAR ASL24660.1 A YRAAHHLLLSHGEAVKLYRSLGLKG. E I . . G ITINIT T AYSAS.DSPODVAAA AOW70162.1 ALQVAHHLLLSHGMAVDIFREEDLPG.EI... G I T INITPAYPAG. DSEKDVKAAS

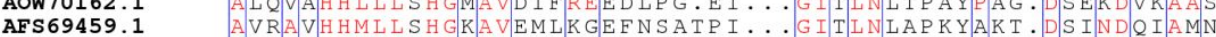
AFO36783.1

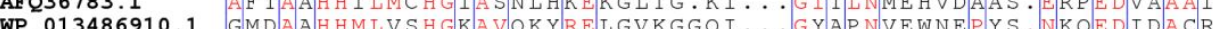
AGS4680.1 ARs 039812498.1 FLKA A E N S VEA A ABQ46970.1 AFRAVHNLLRAHAKAVKVFRET.VKDGKI...GIVFN G G E E AS. E KEE E I RAAR A LA A. HHLNLAHGA.

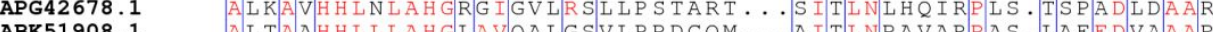
A.LIAAHHLLLAHG LAVQLGSVLPPDCQM...A AT LNPAVARPAS. LAEEDVAAAR

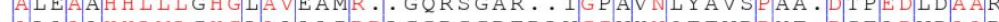
QBC98235.1 A L A A A H L L MLGGLAAAAIRDLGSRSGRTPLVGIVHNQT TVRPYT.DSEADVDAAR ACM59590.1 AMDVVHNIMLSHFKVVKAVKENNIDVEV.... GI T LN LT T PVYF QTERLGYKVSEIE ADD96762.1 $\quad$ GRKAAHHLLLAHGLAMTVLKQNSP T TLN....G IVINET T CYSIS.EDADDIAA TA AXR85509.1 GRTAAHHILLAH LGMKMLKKNVPNAQN....GIVINET T CYSAS.DSPADIAATK ABR73190.1 GRQAAHHILLAHGLALPVIRKNAPKSQV .... G IVINMNRS YAAS. E K TE D Q F A C L EHJ97046.1 A. AAMHHINLAGFGVEASRHVAPKVPV.... GLVINAHSVIPAS.DSDADLKAAE HV348683.1 VLKSTHNLLKAHGAAVKVLRKVAPNT. QL... GIAP TCGVALP I SENKKDIEIARK

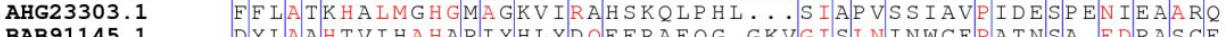
BAB91145.1 DYLAAHTVIHAHARIYHLYDQEFRAEQG. GKVGISININWCEPATNSA. EDRASCE BAI50022.1 DYLTARTVILAHANIYRMYEREFKQQQQ. GKIGISIDNAWCEPISTND.VD. . ACE

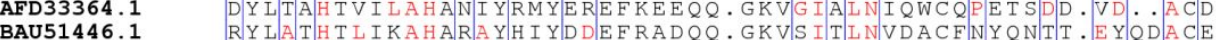
RT PWTVHNITVAHCRAVKVYRDERKDIN. P WI VGHNLLVAHGRAVKVYRDEF KDLN. DGQI G I LN GDF TY PWDSS DP LD REAAE

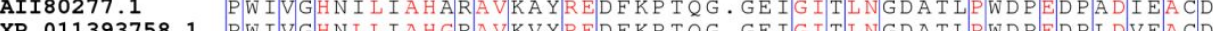

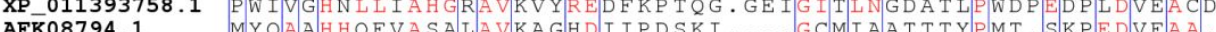
AFK08794.1

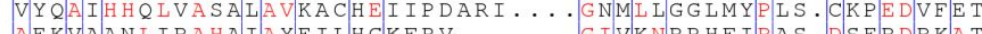

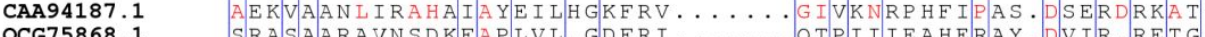

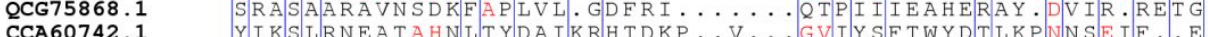
CCA60742.1

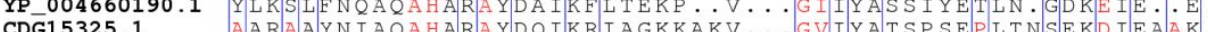

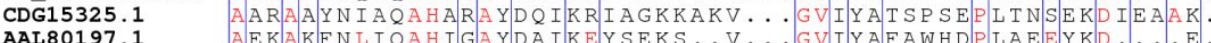
AAL80197.1
consensus $>50$ 


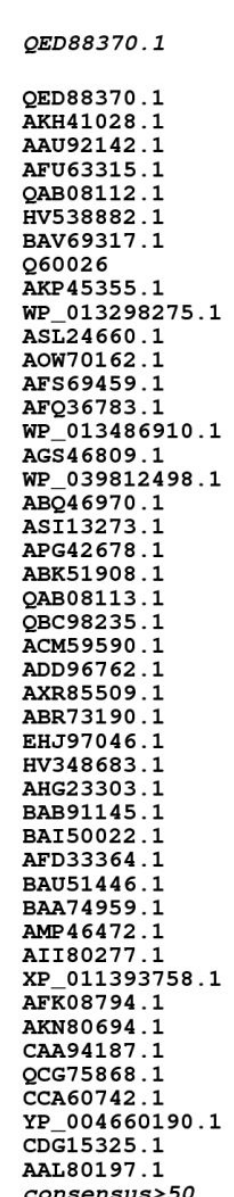

e... .

e.el... eleel ${ }_{260}^{\alpha 11} e_{2 e l e e . . . . . e_{27}}$

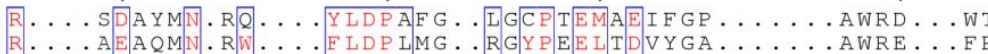

WRD...WT

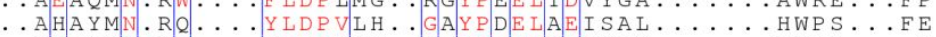

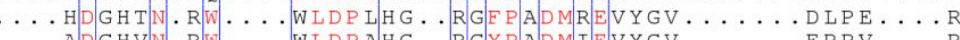
$\ldots$ $\ldots$ FAEPIYN.

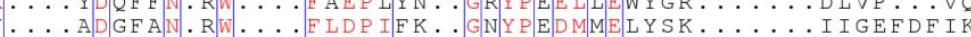
$\cdots \cdots A$ A

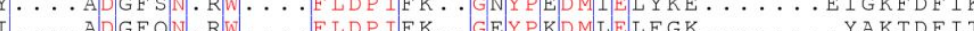

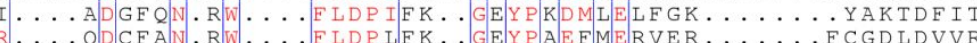
... Q

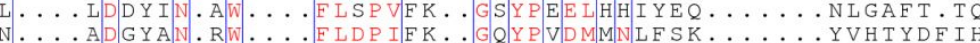

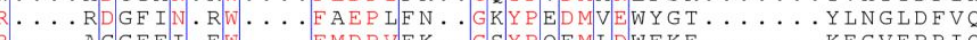

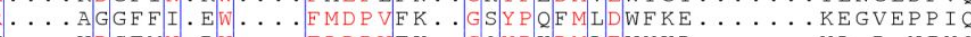

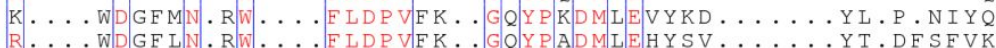

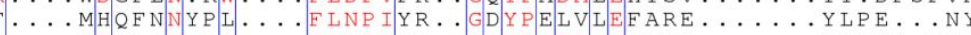

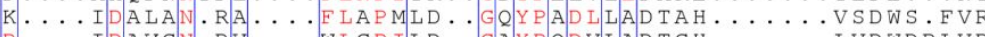

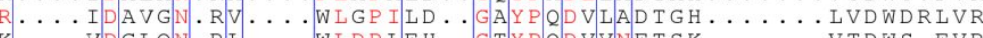

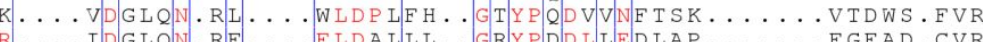

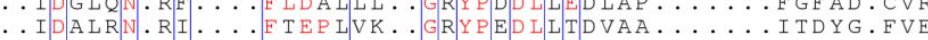

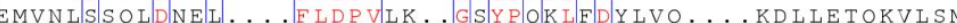
......................

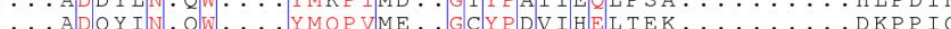

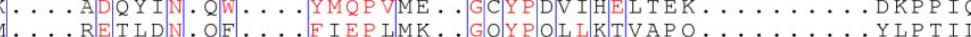
$\ldots$

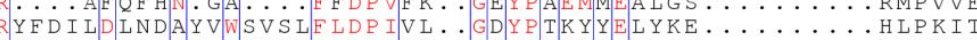

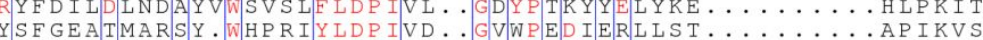

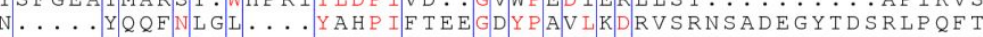

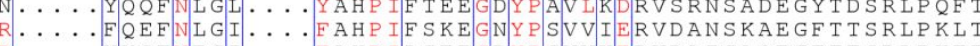

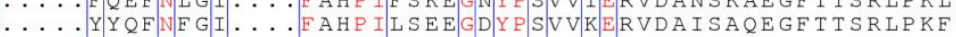
$\ldots$ Q Q Q EMGI....FANP IYSAEGDWPAIVRERVDANSKAEGLAESRLPVF

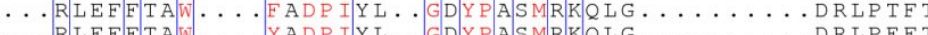

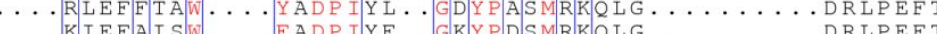

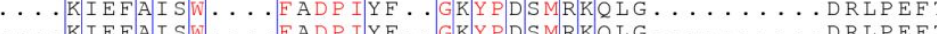

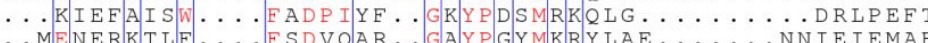

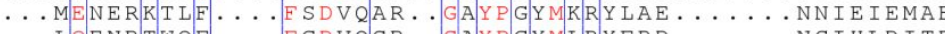

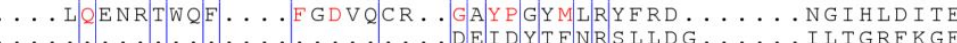

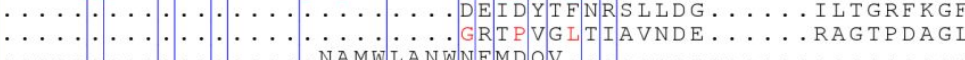

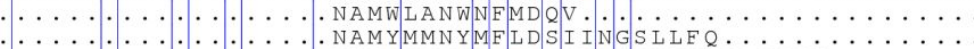

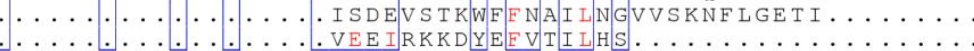

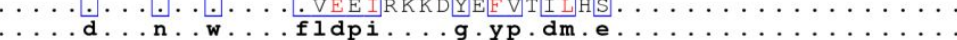

QED88370.1

$\alpha 12$

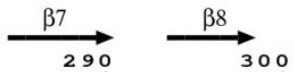

QED 88370.1

AKH 41028.1

AAU92142.1

AFU63315. 1

QAB08112.1

HV538882.1

BAV69317.1

060026

AKP 45355. 1

WP_013298275.1

ASL 24660.1

AOW70162.

AFS69459.1

AFO36783.1

WP_013486910.1

AGS 46809.1

WP_039812498.1

ABQ 46970.1

ASI13273.1

APG42678.

ABK51908.1

QAB08113.1

QBC98235.1

ACM59590.1

ADD 96762.1

AXR85509.1

ABR73190.1

EHJ97046.1

HV348683.1

AHG2 3303.1

BAB91145.1

BAI50022.1

AFD 33364.1

BAU51446.1

BAA74959.1

AMP 46472. 1

AII80277.1

XP_011393758.1

AFK 08794.1

AKN80694.1

CAA94187.1

CAA75868.1

CCA60742.1

YP_004660190.1

CDG15325.

CDG15325.

AAL 80197.1

\section{$280 \quad 290 \quad 300$}

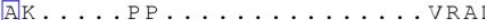

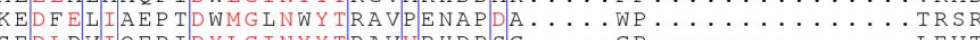

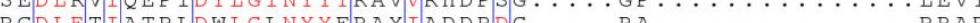
D

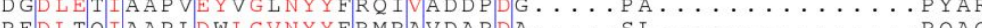

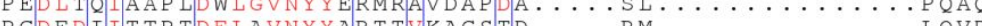
P GDE DI I T TP TDE LAVNYYART TVKAGSTD $\ldots \ldots$ PM $\ldots \ldots \ldots \ldots \ldots$ L LVD

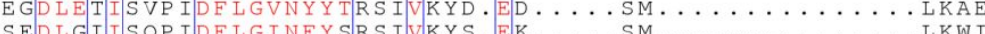
SED LGIIIS QP IDE L INFY SR IVIKYS. EK $\ldots \ldots$ SM $\ldots \ldots \ldots \ldots \ldots$ L LWI DGDLKRIS QKLDELGVNYYTRAVVIKKG.ND $\ldots \ldots$ GI $\ldots \ldots \ldots \ldots \ldots$ LNAE

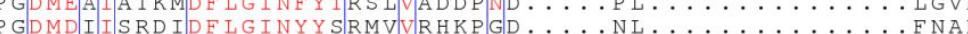

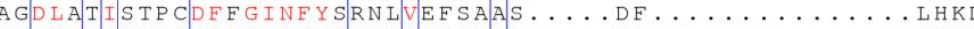
PGDMELI Q QPGDE LG INYYTRS IIIRSTNDA ..... $\ldots \ldots \ldots \ldots \ldots \ldots$ LQVE DGDLEIISQPIDELGINYYTGSVGRYVEDQAAQQHSL . . . . . . . . . FNHE

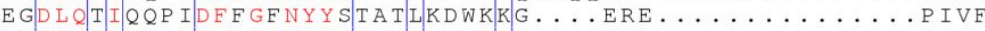
EGDLDTMSAAVDELGINYYSISYITHQPGA...WLE $\ldots \ldots \ldots \ldots \ldots \ldots$ KDDMSEI QEKIDEVGLNYYSGHLVKFDPDA . . . . . . . . . . . . D DINDIVTATPEDIIGVIYYHPVIV . GHYAGSGSRGR DGHGQGTGETWPGCPDI DAPLDMMGVNFYNPSWS ESR NGSDRLPP.D..... EYSPSVGSQH 2 EVK D DMA.

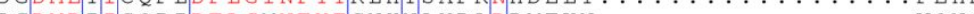
PCDD I AEDSI SQKLDWGLYY

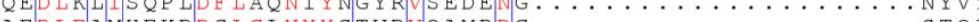
AEDLEAMEKPDSLGLNYYSTR RAMP $\ldots \ldots \ldots \ldots \ldots \ldots \ldots$ AEEVEYIRGTHDELGINFYTALLGKSGVEGYEP.SRYRDSG.......... VILTL SEEVNNTIGTYDEFGLNFYTANLGKDGVEGGIP.SRGRDTG......... AILSQ SEEVTFIKGTYDEMGHNFYTAVMGREGVEGGSP.SRSRDSG............ PDEIEYIRGTYDEFGHNHYTSNYAIPYDGTNDPASDQKDHG........ Y Y LTK PEERALVHGSNDFYGMNHYTSNYIRHRSSPASADDTVGNVD ........... PEEKAFVLGSNDFYGMNHYTSNYIRHRTSPATADDTVGNVD $\ldots \ldots \ldots \ldots . . \cdots$ PEEVALVKGSNDEYGMNHYTANYIKHKTGVPPEDDF LGNLE .......... T LFYN PEEVALVKG SNDFYGMNHY TANYIKHKKGVPPEDDFLGNLE . . . . . T T FYN GDEELIKEHTVDY I GESYYMSMAAS TDPEELAKSGG $\ldots \ldots \ldots \ldots \ldots \ldots \ldots \ldots \ldots \ldots$ NL L

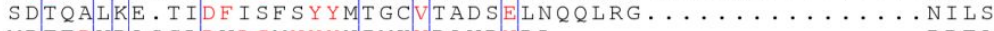

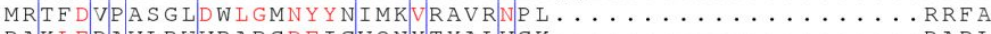

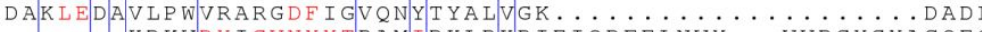
..... KDKVDYI GVNYYTRAMIDKLPRP IEI QDEELNWY . . VVVRGYGYACQEG

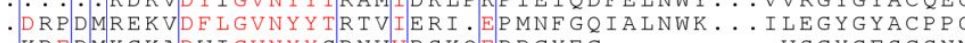
KREDMKGKADWI GVNYYSRNVVRSKQEPPGYEG........... $\ldots \ldots$ KGKLDWIGVNYYSRLVYGAKDGH . LVP . . . . . . . . . . LPGYGFMSERG

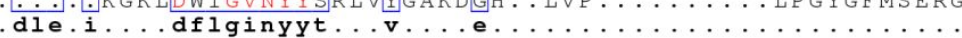




QED88370.1
QED88370.1
AKH41028.1
AAU92142.1
AFU63315.1
QAB08112.1
HV538882.1
BAV69317.1
Q60026
AKP45355.1
WP_013298275.1
ASL24660.1
AOW70162.1
AFS69459.1
AFQ36783.1
WP_013486910.1
AGS46809.1
WP_039812498.1
ABQ46970.1
ASI13273.1
APG42678.1
ABK51908.1
QAB08113.1
QBC98235.1
ACM59590.1
ADD96762.1
AXR85509.1
ABR73190.1
EHJ97046.1
HV348683.1
AHG23303.1
BAB91145.1
BAI50022.1
AFD33364.1
BAU51446.1
BAA74959.1
AMP46472.1
AII80277.1
XP_011393758.1
AFK08794.1
AKN80694.1
CAA94187.1
QCG75868.1
CCA60742.1
YP_004660190.1
CDG15325.1
AAL80197.1
COnsensus>50

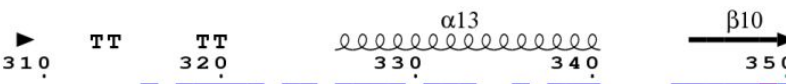
$360^{\mathrm{T}}$

YVRQPGATYTETGW. EVFEQGITETLLWVKERYGD. I PLYVTENGSAFDDPPTA

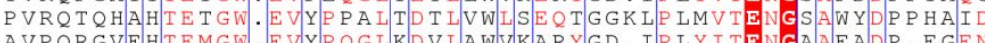
AVP QRGVEHTEMGW. EVYP Q G LIR V LAWVKARYGD. I P LY I T ENGAAFADP . E GEN

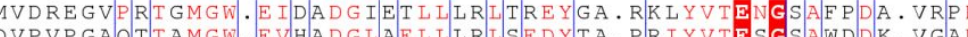
C FVRPPG. FYT AMDW. EVYPOGIYNITNWTHTDYAP.PAIYVTENGAYDDQ . VSA NVPG. PGKRTEMGW. ET SP STY GVEG PGAKTDMGW DMG QIDV.DNEKIEMGN. UY Y C YYS. DY

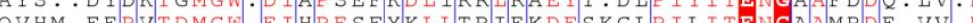
QVHM. EEPVIDMGW. E IHPE SF Y KLLTRI EKDF SKGLP I L I TENGAAMRDE.VV.

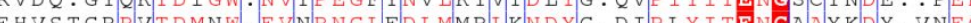
EHVSTGRP VTDMNW. EVNPNG L F L MMRIKNDYG. D I P LY I TENGAAYKDY . VNEQ GHEGGGHRR I SMGW. EVYAKGLSDLIIRLKNDYD. NP VIYVIENGA ADDE. VTN SFVERD LPKTAMGW. EIVPEG IYWI LKKVKEEYN. P P EVYI TENGAAFDDV.VSED E F VQQP GP Y TAMGW. NIEPAGMTELLVSVAT TYP. H QPMMVTENGAAFADEVTVDA AF H LAP GET T AMRW. AVDA S G IYD L LMRIHREHP. GLPMMVTENGA A Y D Y I SPEG QFPEWPERRTAMGW. P IDPSGLYELIIRINRDYP . RP IMITENGAAFDDVVT DNN RFVGQGKPVT GMGW. EVDP D GLIEVLARLHREYP. AVP LYITENGAGYDDVLDADD VEVDPGLPVTAMGW. P IDA T G L YD T LTRLANDYP. GLTLYVTENGAAFDDKL.VDG RWEHPAGEYTEMGW. EVFPQG LYDLLIWIKESYP. Q IPIYITENGAAYNDK . VED P.... TGP LT DMGW. E IYYPKSETELLVTLNN TYT. LPP IFITENGAAMPDS . YNN H... Y Y PRTDIGW. E IHP PALTHLIT SIHQRYA. LPPVYITENGAAMADE . VVD Q.... TVEYTDIGW. EIAPHAFTELIVNIHKQYS. LPPIYITENGAACADQ . I ID QAPAVSDVKTDIGW. EVYAPA LHSLVETLYERYE. LP DCY ITENGACYNMG..VEN PKRKAGYDHTDMGW. P ITP SALYWGPRFICERYN. . LPFYITENGLACHDVVS LDN LEHPPGHPR S GF DW. F IDPEGIYW SVRFHHERYG... LPAYI TENGISG LDWVSEDG

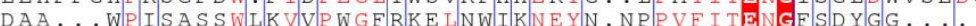
DAA...WP I SASSWLKVPWGR KEINWIKNEYN.NP PVEI DPS ...

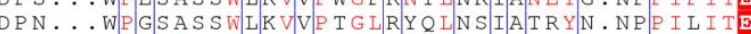
KOGNCIGPETOSPWLRP CAAGFRDFLVWISKRYG. YPPIYVTE KEGQCIGPETES SWIRPCP A GFRDFIVWISKRYN. YPKIYVTE KYGDCI KKGNCI GPETOSFWLRPHAOGFRDLINWISKRYG. YPKIYVTE GVKNPYLK S S E W GW. MVPNPHLPSSEWGW. Q IDP L G LRT L LNMIWDRWQ. . KPVE IVE

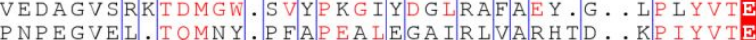
GFALSGRPAASEFGW:

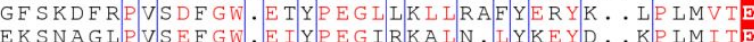
EKSNAGLPVIEEGW. EIYY PEGIRKALN. L Y KEY D . KP LMITE consensus .p.tdmgw.ev.pegl.dll. 1 .eey
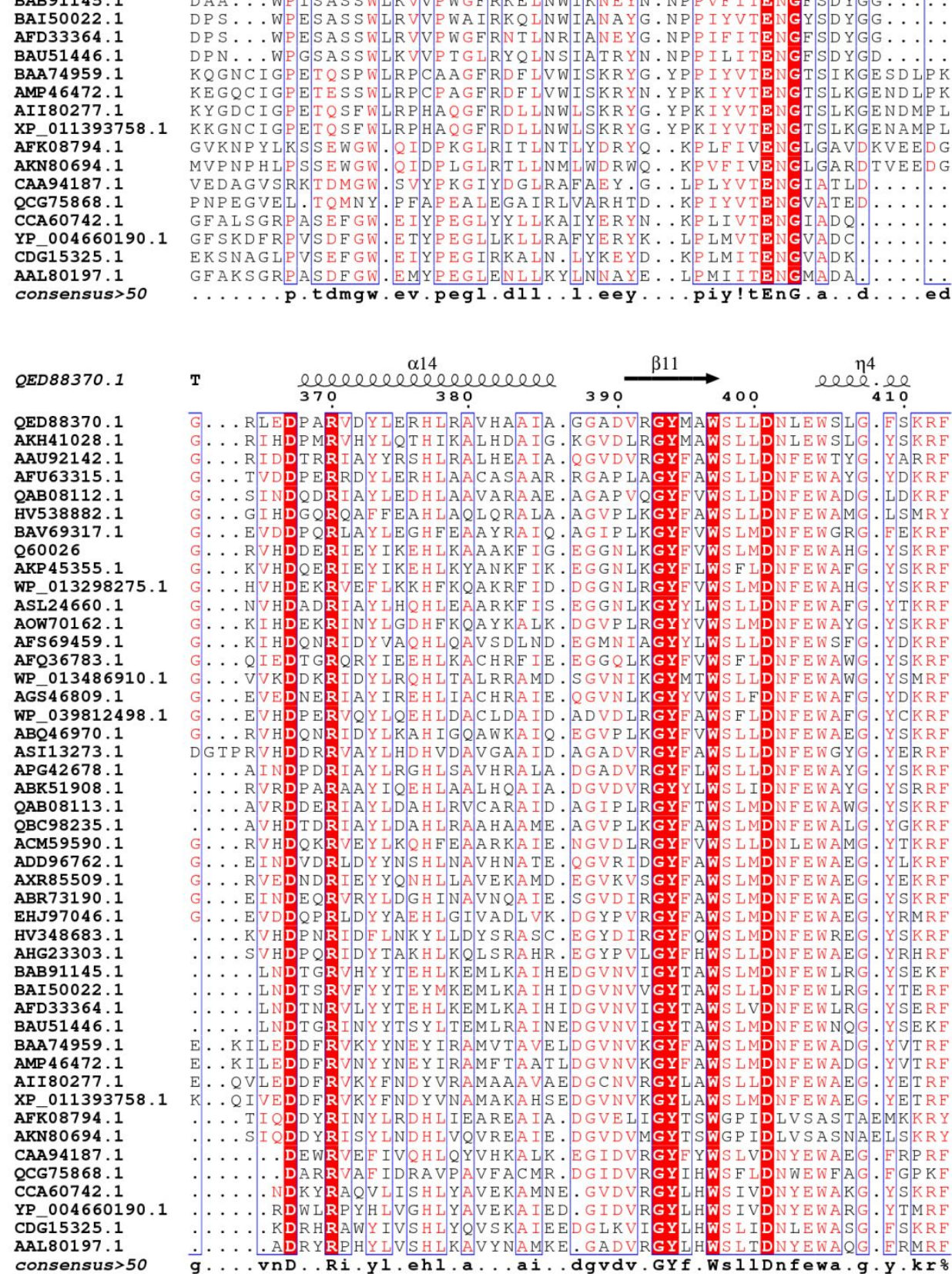


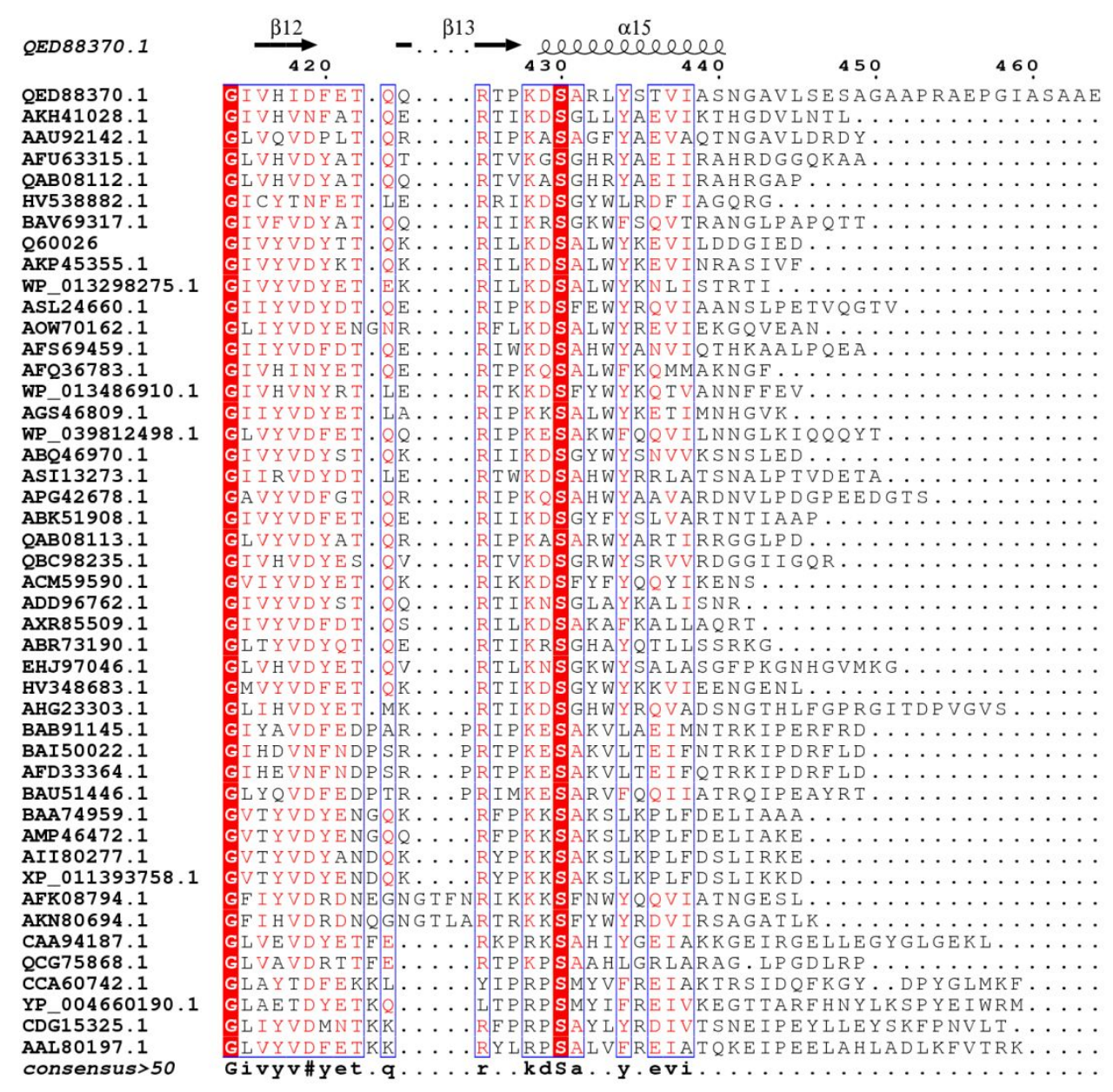

Figure S7. Multiple sequence alignment of Bgl15 with other 45 glucose tolerant

$\beta$-glucosidases from GH1 family. Only the $\beta$-glucosidases with the ability to hydrolyze cellobiose were selected. The alignment was performed by MULTALIN [1], and the secondary structure of Bgl15 was drawn on the top by ESPRIPT [2]. QED88370.1, Bgl15 in this study; AKH41028.1, Bgl6 from a metagenomic library; AAU92142.1, Mbgl from Methylococcus capsulatus; AFU63315.1, Unbgl1A from a metagenomic library; QAB08112.1, AaBGL1 from Actinomadura amylolytica YIM 77502 ${ }^{\mathrm{T}}$; HV538882.1, Td2F2 from a metagenomic library; BAV69317.1, MeBgID2 from a metagenomic library; Q60026, CglT from Thermoanaerobacter brockii; AKP45355.1, BGL from Thermoanaerobacterium aotearoense P8G3\#4; WP_013298275.1, BGL from Thermoanaerobacterium thermosaccharolyticum DSM 571; ASL24660.1, AsBG1 from Alicyclobacillus sp. A4; AOW70162.1, B8CYA8 from Halothermothrix orenii; AFS69459.1, EaBgl1 A from Exiguobacterium antarcticum B7; AFQ36783.1, Gluc1C from Paenibacillus sp. MTCC 5639; 
WP_013486910.1, BcBgl1A from Bacillus cellulosilyticus; AGS46809.1, BglP from Anoxybacillus flavithermus subsp. yunnanensis E13T'; WP_039812498.1, BglD5 from Jeotgalibacillus malaysiensis DSM 28777T; ABQ46970.1, TpBgl1 from Thermotoga petrophila $\mathrm{RKU}-1$; ASI13273.1, CcBgl1B from Cellulosimicrobium cellulans; APG42678.1, Bg10 from a metagenomics library; ABK51908.1, AcBg from Acidothermus cellulolyticus; QAB08113.1, AaBGL2 from Actinomadura amylolytica YIM 77502'; QBC98235.1, ThBGL1A Thermobifida halotolerans YIM 90462"; ACM59590.1, CbBgl1A from Caldicellulosiruptor bescii; ADD96762.1, Bgl1A from a metagenomic library; AXR85509.1, Bgl from Alteromonas sp. L82; ABR73190.1, reBglM1 from Marinomonas sp. MWYL1; EHJ97046.1, H0HC94 from Agrobacterium tumefaciens 5A; HV348683.1, Ks5A7 from a metagenomic library; AHG23303.1, AS-Esc10 from a metagenomic library; BAB91145.1, G1NkBG from Neotermes koshunensis; BAI50022.1, G1mgNtBG1 from Nasutitermes takasagoensis; AFD33364.1, MbmgBG1 from Macrotermes barneyi; BAU51446.1, PaBG1b from Panesthia angustipennis spadica; BAA74959.1, the glucose-tolerant mutant L167W/P172L of TrBg12 (also named Cel1A) from Trichoderma reesei QM9414; AMP46472.1, Thbgl from Trichoderma harzianum; AII80277.1, Bglhi from Humicola insolens; XP_011393758.1, GH1-1 from Neurospora crassa; AFK08794.1, BGL from Bacillus subtilis PS; AKN80694.1, glu1392 from a metagenomics library; CAA94187.1, O08324 from Thermococcus sp.; QCG75868.1, Bgl1317 from a metagenomic library; CCA60742.1, FiBg11A from Fervidobacterium islandicum; YP_004660190, Tt-BGL from Thermotoga thermarum; CDG15325.1, Bgl1 from a metagenomic library; AAL80197.1, PfBgl1 from Pyrococcus furiosus. 


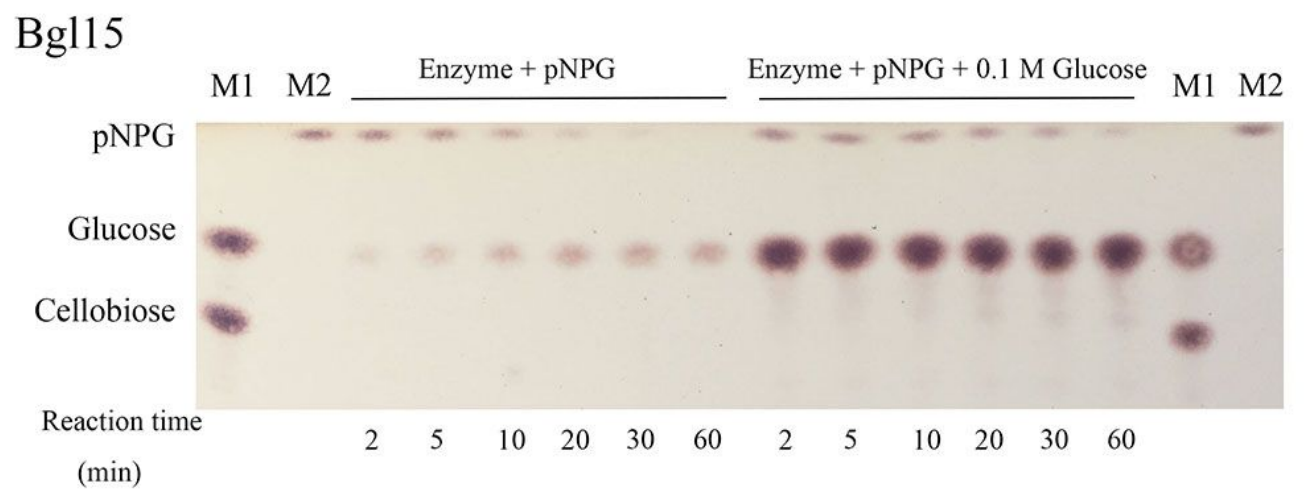

mutant 1R1
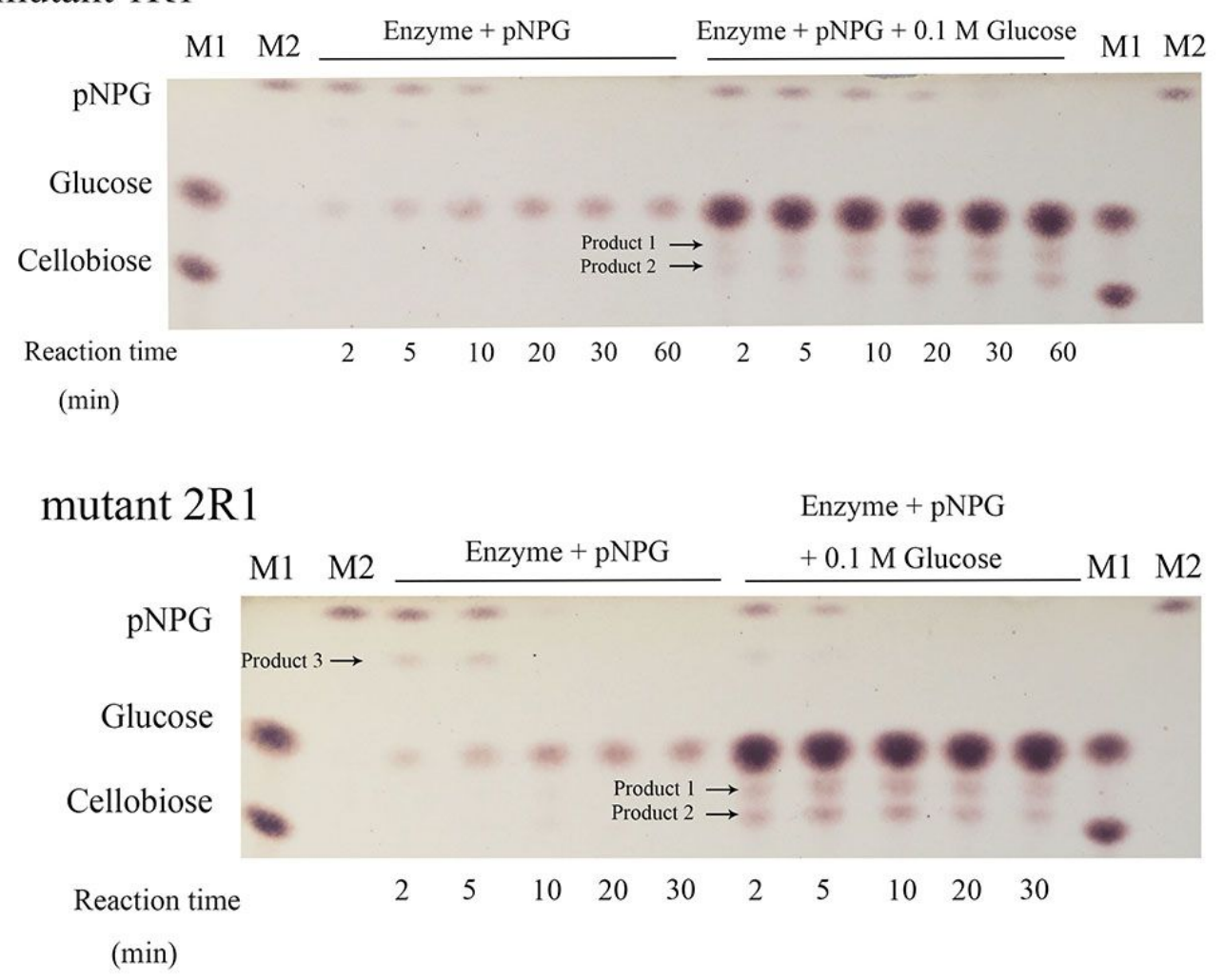

Figure S8. Thin layer chromatography (TLC) analysis of the transglycosylation products of Bgl15 and its mutants. Three transglycosylation products (named product 1, 2 and 3 ) were detected. From its locations on the plates and its appearance in the reactions without glucose, product 3 was presumed to be pNP-Glc-Glc and thus was not related with glucose tolerance. Product 1 and product 2 only appeared in the presence of glucose, and thus may contribute to the improved glucose tolerance of the mutants $1 \mathrm{R} 1$ and 2R1. M1 stranded for Marker, which is the mixture of $0.5 \%(\mathrm{w} / \mathrm{v})$ glucose and $0.5 \%$ cellobiose $(\mathrm{w} / \mathrm{v}) . \mathrm{M} 2$ is a $5 \mathrm{mM}$ pNPG solution. 


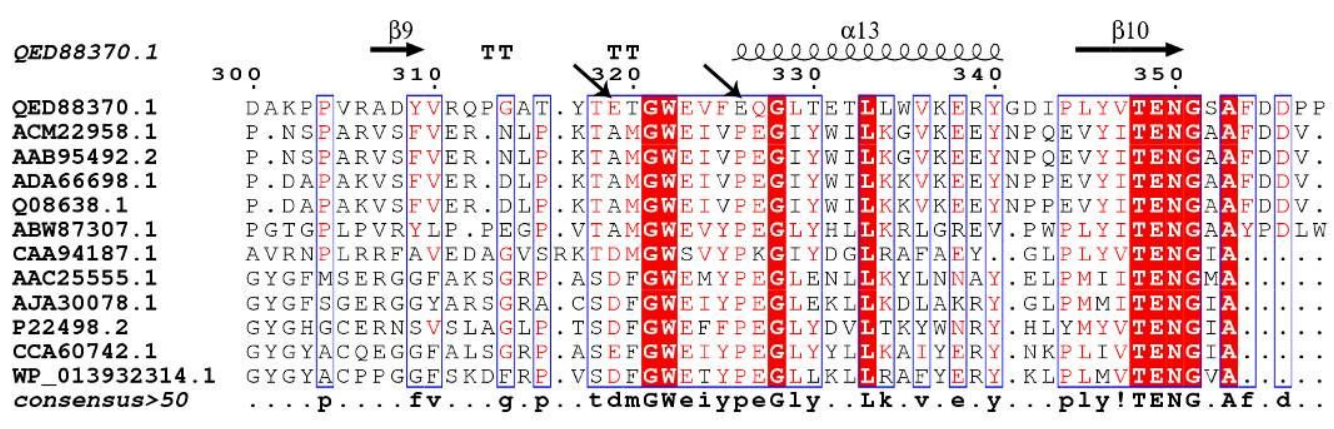

Figure S9. Multiple sequence alignment of Bgl15 with other 11 thermophilic

$\boldsymbol{\beta}$-glucosidases from GH1 family. The alignment was performed by MULTALIN [1], and the secondary structure of Bgl15 was drawn on the top by ESPRIPT [2].

QED88370.1, Bgl15 in this study; ACM22958.1, BGL from Thermotoga neapolitana DSM 4359; AAB95492.2, BGL from Thermotoga neapolitana; ADA66698.1, BGL from Thermotoga naphthophila RKU-10; Q08638.1, BGL from Thermotoga maritima MSB8; ABW87307.1, BGL from Thermus thermophilus; CCA60742.1, BGL from Fervidobacterium islandicum; AAC25555.1, BGL from Pyrococcus furiosus DSM 3638; AJA30078.1, Thermococcus pacificus P-4; P22498.2, BGL from Saccharolobus solfataricus P2; CCA60742.1, BGL from Fervidobacterium islandicum; WP_013932314.1, BGL from Pseudothermotoga thermarum. 


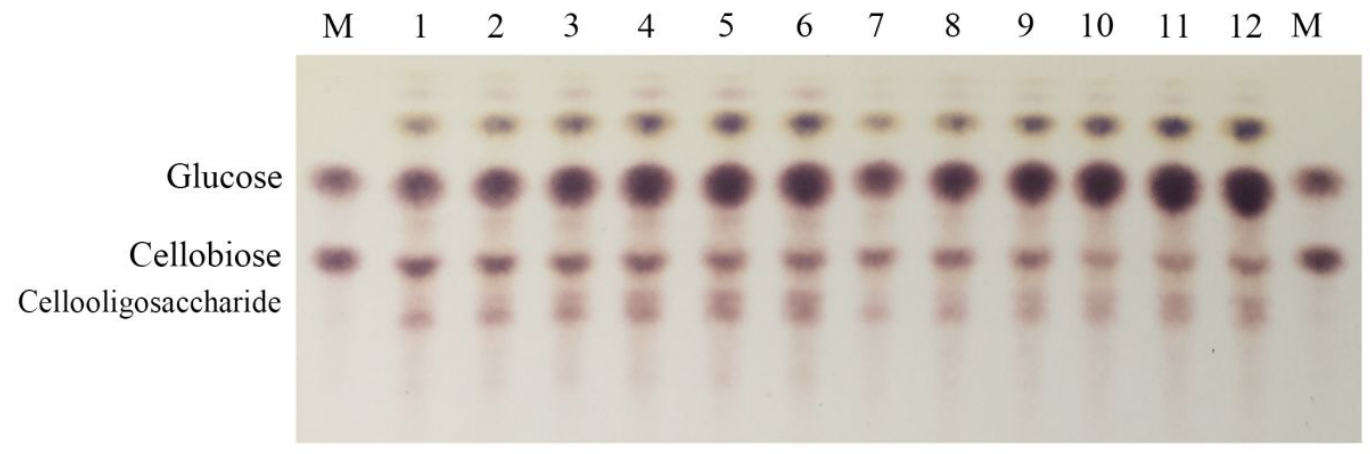

Figure S10. Thin layer chromatography (TLC) analysis of the hydrolysis

products from SCB. M stranded for Marker, which is the mixture of $0.5 \%(\mathrm{w} / \mathrm{v})$ glucose and $0.5 \%$ cellobiose $(\mathrm{w} / \mathrm{v})$. Lane 1-6 represented the results of Celluclast $1.5 \mathrm{~L}$ alone and referred to the hydrolysis time of $12,24,48,72,92$ and $120 \mathrm{~h}$, respectively. Lane 7-14 represented the results of Celluclast 1.5L with mutant 5R1 and referred to the hydrolysis time of $12,24,48,72,92$ and $120 \mathrm{~h}$, respectively. 


\section{Table S1}

Primers used to construct single point mutants in this study.

\begin{tabular}{ll}
\hline Mutants & Primers \\
\hline S39T & FP: CATACCCCCGGGCTGACCGCGAAAGGCG \\
& RP: GACGAAGCGGTGCCAGATGCTCGGCCCTG \\
& FP: CATAGCCCCGGGAACACCGCGAAAGGCG \\
L42N & RP: GACGAAGCGGTGCCAGATGCTCGGCCCTG \\
S167A & FP: GGCGATCACCGACGGCGGCTACCTCCACGG \\
& FP: GTGCATCACCGACGGCGGCTACCTCCACGG \\
V167C & RP: CACGGCTCGTTGAGCGTCGCCCAGCGCTTGAC \\
W178L & FP: CTGGCGCCCGGCCACCGCAATGTCTTC \\
& RP: CGCGCCGTGGAGGTAGCCGCCGTCGG \\
A251L & FP: CAGTACCTCGACCCACTGTTCGGACTCGGC \\
& RP: GCGGTTCATGTACGCGTCCGAGCGCGC \\
E319A & FP: CGCGACCGGCTGGGAAGTCTTCGAGCAAGG \\
& RP: GTGTAGGTCGCTCCCGGCTGGCGGACG \\
E326P & FP: CTTCCCGCAAGGACTGACCGAGACGC \\
& RP: ACTTCCCAGCCGGTCTCGGTGTAGGTCG \\
A396V & FP: GTTTGGTCGTTGCTCGACAATCTCGAATGG \\
& RP: CATGTAGCCGCGGACGTCGGCGCCCC \\
L433F & FP: CTTCTATTCGACGGTCATCGCAAGCAACG \\
& RP: CGAGCGCTGTCCTTCGGCGTTCGCTGCTG \\
\hline
\end{tabular}




\section{Table S2}

Effects of single point mutations on the thermostability of Bgl15.

\begin{tabular}{ll}
\hline Mutants & $\mathrm{T}_{50}$ values $^{\mathrm{a}}\left({ }^{\circ} \mathrm{C}\right)$ \\
\hline 2R1 & 52.9 \\
2R1-V167C & 55.1 \\
2R1-A396V & 55.3 \\
3R1 & 58.1 \\
3R1-E319A & 60.2 \\
3R1-E326P & 60.7 \\
3R1-L433F & 60.5 \\
4R1 & 64.4 \\
4R1-S39T & 65.5 \\
4R1-L42N & 65.9 \\
4R1-A251L & 64.4 \\
\hline
\end{tabular}

a. $\mathrm{T}_{50}$ value was defined as the temperature inactivated $50 \%$ activity in $10 \mathrm{~min}$.

1. Corpet F: Multiple sequence alignment with hierarchical clustering.

Nucleic Acids Research 1988, 16(22):10881-10890.

2. Robert X, Gouet P: Deciphering key features in protein structures with the new ENDscript server. Nucleic Acids Res 2014, 42(Web Server issue):W320-324. 